\title{
Cardiopulmonary Resuscitation Performance may Improve with Consideration of Performer's Characteristics
}

\author{
Hikaru Nachi ${ }^{1,2}$, Sho Nachi ${ }^{3}$, Hideshi Okada ${ }^{3^{*}}$, Kodai Suzuki ${ }^{3}$, Takahito Miyake ${ }^{3}$, Takahiro Yoshida ${ }^{3}$, Shinji Ogura $^{3}$ and Eiichi Chihara ${ }^{1,2}$ \\ ${ }^{1}$ Division of General Medicine,Department of Anesthesiology, Asahi University School of Dentistry, Mizuho, Japan \\ ${ }^{2}$ Department of Anesthesiology, Asahi University Murakami Memorial Hospital, Gifu, Japan
}

${ }^{3}$ Department of Emergency and Disaster Medicine, Gifu University Graduate School of Medicine, Gifu, Japan

\begin{abstract}
Background: In basic life support, chest compressions are a very important and basic skill. Acquiring the ability to perform chest compressions is affected by individual characteristics such as physique and physical strength, although all persons can learn to Perform Cardiopulmonary Resuscitation (CPR), including chest compressions. The aim of this study was to examine differences in physique between males and females and Basic Life Support (BLS) skill using a portable manikin with automated corrective feedback.
\end{abstract}

Methods: Participants were 120 fourth-year preclinical dental school students (87 males, 33 females). For skill assessment, students performed chest compressions and single rescuer CPR using a Laerdal Resusci Anne Skill Reporter TM manikin for 2 minutes each before and after CPR. Outcome measures were (1) compression depth ( $\mathrm{mm}$ ), (2) compression rate (number of compressions per minute), (3) compression release depth (recoil, $\mathrm{mm}$ ), and (4) hand position before and after the BLS course.

Results: After the BLS course, compression depth and rate improved significantly. Both before and after the BLS course, male students performed deeper chest compressions than female students. In females, the duration of acceptable chest compressions was significantly shorter than in males.

Conclusion: CPR performance was significantly different between males and females, probably due to differences in physique. Therefore, it is necessary for individualized instruction adapted to the learner's characteristics.

Keywords: Resuscitation; Basic life support; Chest compression

\section{Introduction}

Basic Life Support (BLS) is a level of medical care provided for victims of life-threatening illnesses or injuries until they can receive comprehensive medical care at a hospital. It can be provided by trained medical personnel, including emergency medical technicians and paramedics, and by qualified bystanders. Therefore, it is very important to acquire BLS skills. However, studies have documented that the quality of cardiopulmonary resuscitation (CPR) even by nurses and physicians is often poor, even when they have been trained in CPR [1-6].

It has been reported that the acquisition of chest compression skills is affected by individual characteristics such as physique and physical strength, but all persons are capable of learning CPR, including chest compression [7-9]. In particular, differences in physique between males and females is very important; a previous study showed that chest compression quality over 15 minutes performed by females was lower compared with males [10]. Therefore, to ensure that CPR is performed appropriately, individualized instruction adapted to the learner's characteristics is necessary. However, few studies have examined the acquisition of CPR skills.

The recent development of a new device that can objectively evaluate BLS skills [11] leads to the possibility of more effective BLS instruction. The aim of this study was to examine differences in physique between males and females and BLS skill using a portable manikin with automated corrective feedback.

\section{Methods}

\section{Participants}

The participants were 120 fourth-year preclinical dental school students ( 87 males, 33 females). This study was approved by the university's Institutional Review Board. Each student provided written consent for study participation. Before the study, all of them attended a BLS course by the Japanese Association for Acute Medicine. At first, instruction for chest compression was performed using a practicewhile-watching method. Next, instruction included scenario training if a maneuver was insufficient.

\section{Assessment of CPR psychomotor skills}

For the skills assessment, students performed compressions and single rescuer CPR using a Laerdal Resusci Anne Skill Reporter TM manikin before and after CPR for 2 minutes each. Outcome measures were (1) compression depth ( $\mathrm{mm}),(2)$ compression rate (number of compressions per minute), (3) compression release depth (recoil, $\mathrm{mm}$ ), and (4) hand position. The acceptable range of CPR was 1) a single minimum compression depth of $\geq 50 \mathrm{~mm}, 2$ ) an optimal target range of 100 and 120 compressions per minute, and 3) hand position on the lower half of the sternum, based on American Heart Association guidelines [12].

*Corresponding author: Hideshi Okada, MD, PhD, Department of Emergency and Disaster Medicine, Gifu University Graduate School of Medicine, 1-1 Yanagido, Gifu 501-1194, Japan, Tel: +81582306448; E-mail: hideshi@gifu-u.ac.jp

Received December 30, 2017; Accepted January 05, 2018; Published January 10, 2018

Citation: Nachi H, Nachi S, Okada H, Suzuki K, Miyake T, et al. (2018) Cardiopulmonary Resuscitation Performance may Improve with Consideration of Performer's Characteristics. J Health Educ Res Dev 6: 244. doi: 10.4172/23805439.1000244

Copyright: (c) $2018 \mathrm{Nachi} \mathrm{H}$, et al. This is an open-access article distributed under the terms of the Creative Commons Attribution License, which permits unrestricted use, distribution, and reproduction in any medium, provided the original author and source are credited. 
(A)

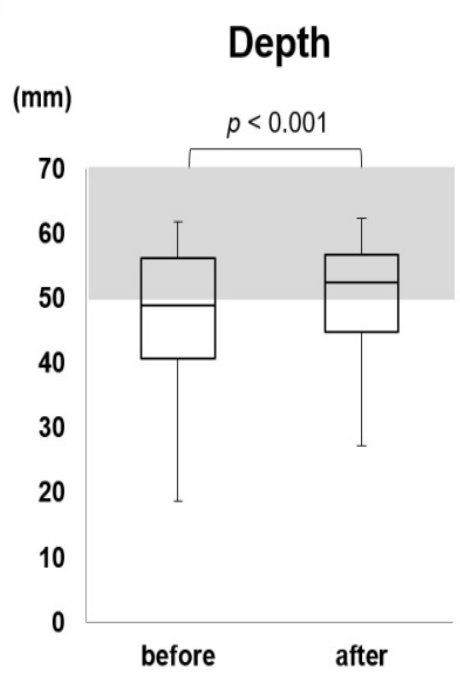

(B)

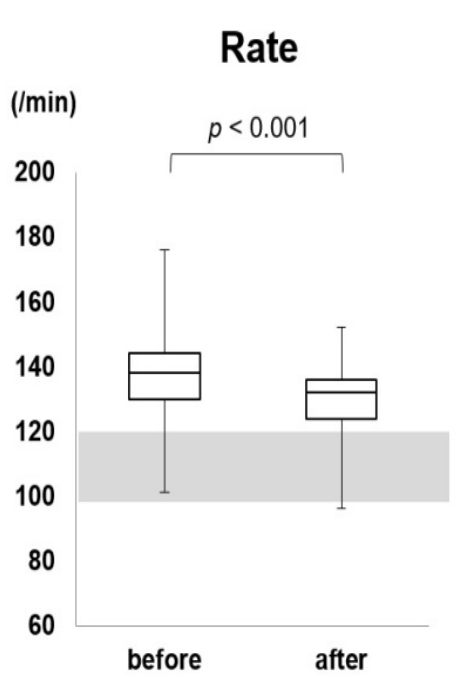

(C)

\section{Recoil}

$(\mathrm{mm})$

$p=0.115$

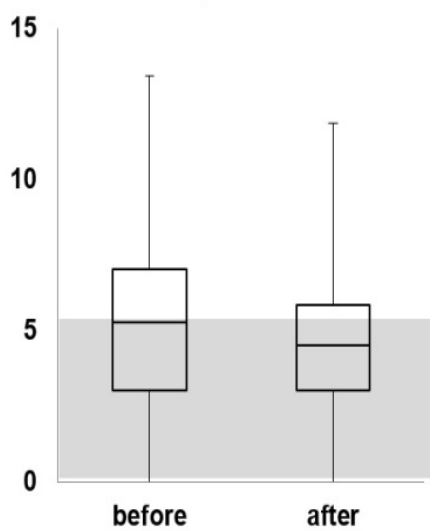

Figure 1: Chest Compression Depth, Rate, and Recoil Before and After the BLS Course. (A) Chest compression depth (mm), (B) chest compression rate (number of compressions per minute), (C) chest compression release depth (recoil, $\mathrm{mm}$ ). The gray zone indicates the acceptable range for CPR. The compression depth and rate significantly improved after the BLS course. There was no significant difference in recoil before and after the BLS course. BLS: Basic Life Support; CPR: Cardiopulmonary Resuscitation.

(A)

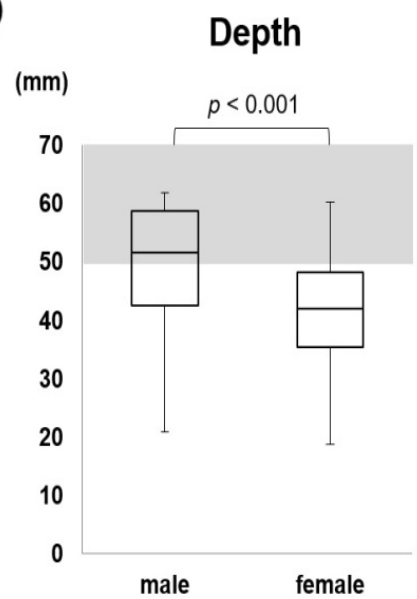

(B)

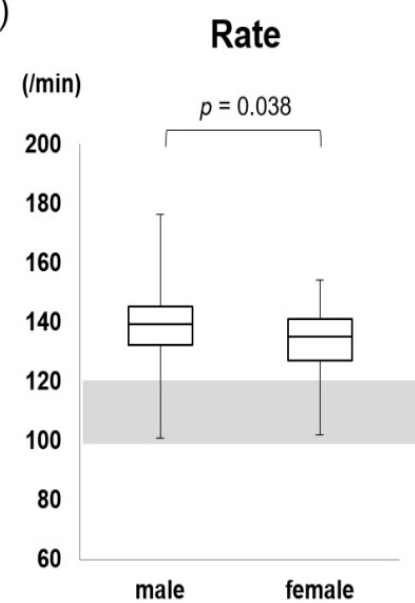

(C)

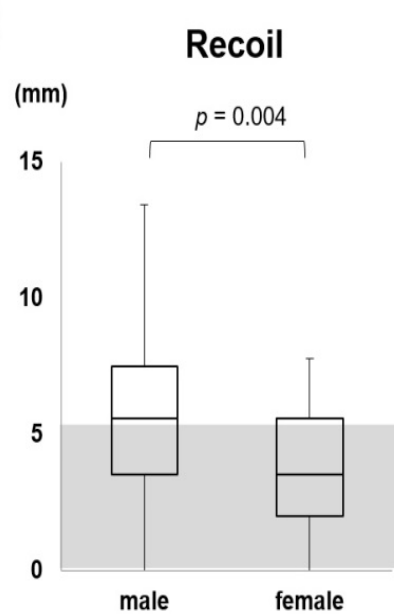

Figure 2: Chest Compression Depth, Rate, and Recoil by Sex Before the BLS Course. (A) Chest compression depth (mm), (B) chest compression rate (number of compressions per minute), (C) chest compression release depth (recoil, $\mathrm{mm}$ ). The gray zone indicates the acceptable range for CPR. BLS: Basic Life Support; CPR: Cardiopulmonary Resuscitation.

\section{Data analysis}

Data were summarized as means (standard deviation) for continuous variables and frequencies (\%) for categorical variables. Differences between groups were evaluated using the Wilcoxon signedrank test, Mann-Whitney U test, or Spearman' rank-order correlation as appropriate; $\mathrm{p}<0.05$ was considered to indicate statistical significance.

\section{Results}

\section{Characteristics of study participants}

Table 1 summarizes the characteristics of the study participants. There were no significant differences in age between males and females. Males had significantly higher height, weight, and Body Mass Index (BMI) than females.
Hand position, compression depth, compression rate, and recoil

Appropriate performance of each skill was assessed during 2 minutes of CPR. Regarding hand position, all participants used the correct

\begin{tabular}{|c|c|c|c|}
\hline \multirow{2}{*}{ Sex $(\mathbf{n})$} & Male & Female & \multirow{2}{*}{ p value } \\
\cline { 2 - 4 } & $\mathbf{8 7}$ & $\mathbf{3 3}$ & \\
\hline Age (years) & $24(22-25)$ & $23(22-25)$ & 0.38 \\
\hline Height $(\mathrm{cm})$ & $170(166-174.5)$ & $158(155-161)$ & $<0.001$ \\
\hline Weight $(\mathrm{kg})$ & $69(62-81.5)$ & $53.3(46-62.1)$ & $<0.001$ \\
\hline BMI $\left(\mathrm{kg} / \mathrm{m}^{2}\right)$ & $24(21-26.6)$ & $20.1(19.3-22.3)$ & $<0.001$ \\
\hline
\end{tabular}

Values are medians (interquartile range); BMI: Body Mass Index. Table 1: Characteristics of the Study Participants. 
(A)

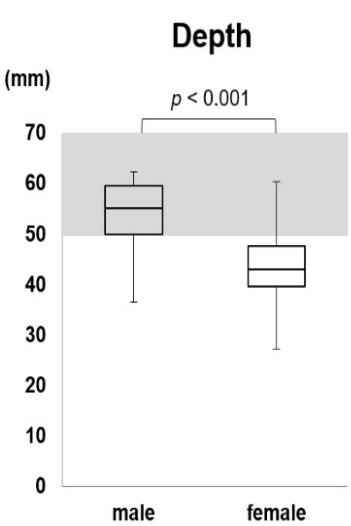

(B)

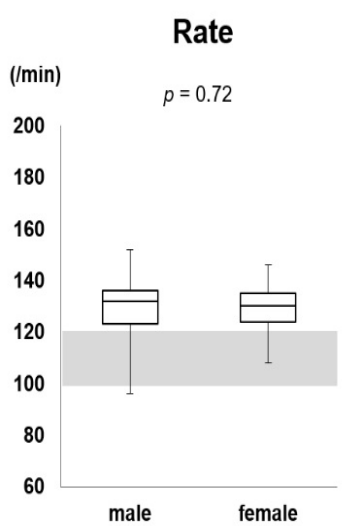

(C) Recoil

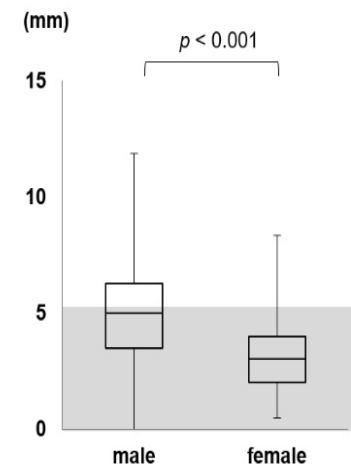

Figure 3: Chest Compression Depth, Rate, and Recoil by Sex after the BLS Course. (A) Chest compression depth (mm), (B) chest compression rate (number of compressions per minute), (C) chest compression release depth (recoil, $\mathrm{mm}$ ). The gray zone indicates the acceptable range for CPR. BLS: Basic Life Support; CPR: Cardiopulmonary Resuscitation.

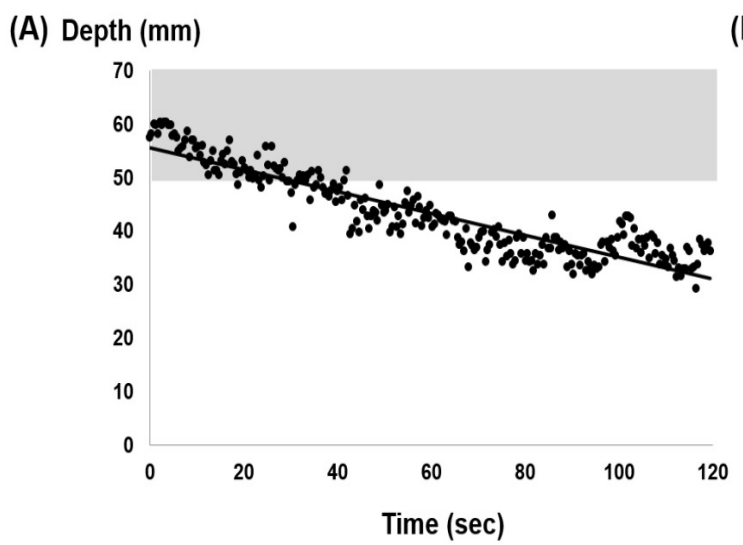

(B) Time (sec)

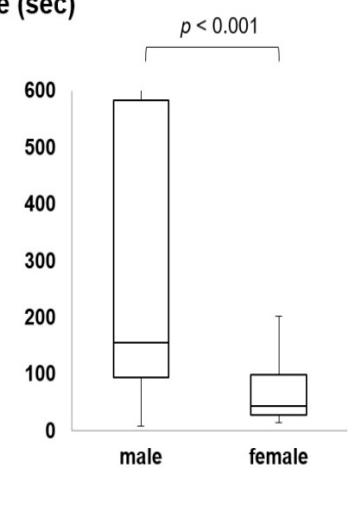

Figure 4: Changes in Chest Compression Over Time. (A) A representative case in which chest compression depth gradually became shallower over 2 minutes after the BLS course. (B) Comparison of the duration of acceptable chest compressions between males and females. In males, the duration was 155 (93-583) seconds compared to 44 (28-98) seconds in females. BLS: Basic Life Support; CPR: Cardiopulmonary Resuscitation.

hand position after the BLS course. After the BLS course, compression depth was significantly deeper than before the course (Figure 1A). Although the compression rate was faster than the acceptable range of 100 to 120 compressions per minute before and after the BLS course, it was significantly slower and closer to the acceptable range after the BLS course (Figure 1B). There were no significant differences in recoil before and after the BLS course (Figure 1C).

\section{Comparisons by gender}

Before the BLS course, males performed significantly deeper compressions than females (Figure 2A). The compression rate and depth of recoil were slower and shallower for females than for males (Figure 2B and 2C). After the BLS course, males had significantly higher compression depth than females (Figure 3A). Recoil depth was shallower for females than for males (Figure $3 \mathrm{~B}$ ). There were no significant differences in compression rate between males and females (Figure 3C).

\section{Correlation between chest compression characteristics and physique after the BLS course}

In males, compression depth and recoil were weakly but significantly correlated with height and weight (Table 2A). On the other hand, compression depth was significantly correlated with height in females $(\mathrm{r}=0.63, \mathrm{p}<0.001)$ (Table $2 \mathrm{~B})$. In addition, compression depth was weakly but significantly correlated with weight in females $(r=0.37$, $\mathrm{p}<0.05)$

\section{Change in chest compressions over 2 minutes after the BLS course}

Chest compression depth gradually became shallower over time for $96.7 \%$ of study participants (Figure $4 \mathrm{~A}$ ). Compression recoil and rate did not change over time. Among the 116 students (83 males and 33 females) for whom chest compression depth gradually became more shallow, there were 88 students ( 73 males and 15 females) who performed acceptable chest compressions at the start. There were 48 students (45 males and 3 females) who performed acceptable chest compressions continuously for 2 minutes.

The group that initially performed acceptable chest compressions but whose chest compression depth became shallower was further investigated. Females had a significantly shorter duration of acceptable chest compressions than males (Figure 4B). 
(A) Males

\begin{tabular}{|c|c|c|c|}
\hline & Age (years) & Height (cm) & Weight (kg) \\
\hline Depth (mm) & -0.029 & $0.287^{* *}$ & $0.249^{*}$ \\
\hline Rate $(\mathrm{cpm})$ & 0.014 & -0.085 & -0.094 \\
\hline Recoil $(\mathrm{mm})$ & 0.116 & $0.268^{*}$ & $0.287^{* *}$ \\
\hline${ }^{*} \mathrm{p}<0.05 ;{ }^{* *} \mathrm{p}<0.01$ & & & \\
\hline
\end{tabular}

\section{(B) Females}

\begin{tabular}{|c|c|c|c|}
\hline & Age (years) & Height (cm) & Weight (kg) \\
\hline Depth $(\mathrm{mm})$ & -0.045 & $0.63^{\star \star *}$ & $0.365^{*}$ \\
\hline Rate $(\mathrm{cpm})$ & 0.103 & -0.186 & -0.057 \\
\hline Recoil $(\mathrm{mm})$ & -0.152 & 0.139 & 0.222 \\
\hline
\end{tabular}

${ }^{*} p<0.05,{ }^{* *} p<0.01,{ }^{* * *} p<0.001$; BLS: Basic Life Support; cpm: Compressions Per Minute

Department of Anesthesiology,

Table 2: Correlation between Physique and Chest Compression Performance after a BLS Course.

\section{Discussion}

The present study assessed CPR performance before and after a BLS course using a CPR simulator that is able to objectively evaluate BLS skill. After the BLS course, the percentage of correct chest compressions improved. Therefore, the BLS course was useful in CPR education for dental students. Chest compression depth was 40.7-56.2 $\mathrm{mm}$ before the BLS course and 44.8-56.7 mm after BLS course. On the other hand, chest compression recoil was 3.0-7.0 $\mathrm{mm}$ before the BLS course and 3.0-5.8 $\mathrm{mm}$ after the BLS course. A previous report showed that chest compression depth was more important than recoil [13]. The current study showed that BLS instruction improved compression depth rather than recoil. Therefore, the current study focused on chest compression depth.

Before and after the BLS course, male students performed deeper chest compressions than female students. This result was consistent with a previous report that chest compression quality is lower for females compared with males over 15 minutes of chest compressions [10]. In female students, there was a correlation between chest compression depth and height, but there was no correlation between depth and physique in male students. Chest compressions were performed with bending at the hip joint and involved the upper arm muscles, pectoralis major, erector spinae, and thigh muscles $[14,15]$. Therefore, to achieve effective chest compression depth, more muscle activity is required in a short person because of the lower center of gravity. Moreover, antagonized muscle (e.g., spine) activity is required to maintain a forward-bent posture when performing chest compressions [14]. Although physical strength measurements were not performed in the current study, it has been previously reported that there is a positive correlation between high-quality chest compressions and physical strength $[10,16]$. Since short female students have lower muscle strength and need more muscle activity to perform chest compressions, it is difficult to continue high-quality chest compressions because muscle fatigue occur earlier [16]. In the present study, a trend of chest compression depth becoming shallower over time was observed. This study showed that the duration of effective chest compressions performed by female students was significantly shorter compared with male students, which is consistent with previous reports $[14,16$ 20]. Although American Heart Association guidelines recommend changing the person performing chest compressions every 1-2 minutes, [17] differences by sex, physique, or other characteristics were not described. To ensure high-quality chest compressions, more frequent changes might be needed.
In conclusion, the present study showed CPR performance varied significantly between males and females, which were probably due to differences in physique. Therefore, individualized instruction adapted to the learner's characteristics is necessary.

\section{Study Limitation}

Since the sample size was small, further study is required. In the current study, chest compression was performed on springs inside a manikin, which compress in a linear fashion. However, during actual clinical conditions, chest compressions are non-linear, which is a substantial difference between the conditions in this study and actual clinical practice [21].

\section{References}

1. Abella BS, Alvarado JP, Myklebust H, Edelson DP, Barry A, et al. (2005) Quality of cardiopulmonary resuscitation during in-hospital cardiac arrest. JAMA 293 305-310.

2. Abella BS, Sandbo N, Vassilatos P, Alvarado JP, O'Hearn N, et al. (2005) Chest compression rates during cardiopulmonary resuscitation are suboptimal: a prospective study during in-hospital cardiac arrest. Circulation 111: 428-434.

3. Aufderheide TP, Lurie KG (2004) Death by hyperventilation: a common and life-threatening problem during cardiopulmonary resuscitation. Crit Care Med 32: S345-351.

4. Dine CJ, Gersh RE, Leary M, Riegel BJ, Bellini LM, et al (2008) Improving cardiopulmonary resuscitation quality and resuscitation training by combining audiovisual feedback and debriefing. Crit Care Med 36: 2817-2822.

5. Nyman J, Sihvonen M (2000) Cardiopulmonary resuscitation skills in nurses and nursing students. Resuscitation 47: 179-184.

6. Wik L, Kramer-Johansen J, Myklebust H, Sorebo H, Svensson L, et al. (2005) Quality of cardiopulmonary resuscitation during out-of-hospital cardiac arrest JAMA 293: 299-304.

7. Ashton A, McCluskey A, Gwinnutt CL, Keenan AM (2002) Effect of rescue fatigue on performance of continuous external chest compressions over $3 \mathrm{~min}$ Resuscitation 55: 151-155.

8. Gasco C, Avellanal M, Sanchez M (2000) Cardiopulmonary resuscitation training for students of odontology: skills acquisition after two periods of learning. Resuscitation 45: 189-194

9. Hong DY, Park SO, Lee KR, Baek KJ, Shin DH (2012) A different rescue changing strategy between 30:2 cardiopulmonary resuscitation and hands-only cardiopulmonary resuscitation that considers rescuer factors: a randomised cross-over simulation study with a time-dependent analysis. Resuscitation 83 353-359.

10. Hansen D, Vranckx P, Broekmans T, Eijnde BO, Beckers W, et al. (2012) Physical fitness affects the quality of single operator cardiocerebral resuscitation in healthcare professionals. Eur J Emerg Med 19: 28-34.

11. Niles D, Sutton RM, Donoghue A, Kalsi MS, Roberts K, et al. (2009) "Rolling Refreshers": a novel approach to maintain CPR psychomotor skill competence. Resuscitation 80: 909-912

12. Meaney PA, Bobrow BJ, Mancini ME, Christenson J, de Caen AR, et al. (2013) Cardiopulmonary resuscitation quality: [corrected] improving cardiac resuscitation outcomes both inside and outside the hospital: a consensus statement from the American Heart Association. Circulation 128: 417-435.

13. Glatz AC, Nishisaki A, Niles DE, Hanna BD, Eilevstjonn J, et al. (2013) Sterna wall pressure comparable to leaning during CPR impacts intrathoracic pressure and haemodynamics in anaesthetized children during cardiac catheterization. Resuscitation 84: 1674-1679.

14. Trowbridge C, Parekh JN, Ricard MD, Potts J, Patrickson WC, et al. (2009) A randomized cross-over study of the quality of cardiopulmonary resuscitation among females performing 30:2 and hands-only cardiopulmonary resuscitation. BMC Nurs 8: 6.

15. Yasuda Y, Kato Y, Sugimoto K, Tanaka S, Tsunoda N, et al. (2013) Muscles used for chest compression under static and transportation conditions. Prehosp Emerg Care 17: 162-169. 
Citation: Nachi H, Nachi S, Okada H, Suzuki K, Miyake T, et al. (2018) Cardiopulmonary Resuscitation Performance may Improve with Consideration of Performer's Characteristics. J Health Educ Res Dev 6: 244. doi: 10.4172/2380-5439.1000244

Page 5 of 5

16. Ock SM, Kim YM, Chung J, Kim SH (2011) Influence of physical fitness on the performance of 5-minute continuous chest compression. Eur J Emerg Med 18: 251-256.

17. Berg RA, Hemphill R, Abella BS, Aufderheide TP, Cave DM, et al. (2010) Part 5: adult basic life support: 2010 American Heart Association Guidelines for Cardiopulmonary Resuscitation and Emergency Cardiovascular Care. Circulation 122: S685-705.

18. Foo NP, Chang JH, Lin HJ, Guo HR (2010) Rescuer fatigue and cardiopulmonary resuscitation positions: A randomized controlled crossover trial. Resuscitation 81: $579-584$
19. Sugerman NT, Edelson DP, Leary M, Weidman EK, Herzberg DL, et al. (2009) Rescuer fatigue during actual in-hospital cardiopulmonary resuscitation with audiovisual feedback: a prospective multicenter study. Resuscitation 80: 981-984.

20. McDonald CH, Heggie J, Jones CM, Thorne CJ, Hulme J (2013) Rescuer fatigue under the 2010 ERC guidelines, and its effect on cardiopulmonary resuscitation (CPR) performance. Emerg Med J 30: 623-627.

21. Tomlinson AE, Nysaether J, Kramer-Johansen J, Steen PA, Dorph E (2007) Compression force-depth relationship during out-of-hospital cardiopulmonary resuscitation. Resuscitation 72: 364-370. 